\title{
Body Weight Gain Original Result Unit
}

National Cancer Institute

\section{Source}

National Cancer Institute. Body Weight Gain Original Result Unit. NCI Thesaurus. Code C119768.

The unit of measure for the result of the body weight gain assessment as originally received or collected. 